\title{
EEG Motor-Imagery BCI System Based on Maximum Overlap Discrete Wavelet Transform (MODWT) and Machine learning algorithm
}

\author{
Samaa S. Abdulwahab*, Hussain K. Khleaf, and Manal H. Jassim \\ Electrical Engineering Department, University of Technology, Iraq, Baghdad
}

\author{
Correspondence \\ * Samaa S. Abdulwahab \\ Department of Electrical Engineering, \\ University of Technology, Baghdad, Iraq. \\ Email: $\underline{\text { 316393@ @student.uotechnology.edu.iq }}$
}

\begin{abstract}
The ability of the human brain to communicate with its environment has become a reality through the use of a Brain-Computer Interface (BCI)-based mechanism. Electroencephalography (EEG) has gained popularity as a non-invasive way of brain connection. Traditionally, the devices were used in clinical settings to detect various brain diseases. However, as technology advances, companies such as Emotiv and NeuroSky are developing low-cost, easily portable EEG-based consumer-grade devices that can be used in various application domains such as gaming, education. This article discusses the parts in which the EEG has been applied and how it has proven beneficial for those with severe motor disorders, rehabilitation, and as a form of communicating with the outside world. This article examines the use of the SVM, $k-N N$, and decision tree algorithms to classify EEG signals. To minimize the complexity of the data, maximum overlap discrete wavelet transform (MODWT) is used to extract EEG features. The mean inside each window sample is calculated using the Sliding Window Technique. The vector machine (SVM), $k$-Nearest Neighbor, and optimize decision tree load the feature vectors.
\end{abstract}

KEYWORDS: EEG, BCI, Motor imagery, MODWT, SVM, k-NN, Decision Tree, EMOTIV EPOC+

\section{INTRODUCTION}

The last two decades have seen a rise in research on BrainComputer Interface (BCI) applications[1]. Nowadays, MI EEG-based BCI is a promising technology due to its enormous domain in both medical and non-medical implementations. The MI task is accomplished by imagining performing a specific task without actually performing it [2]. The widely used MI tasks in researches are the imaginations of the right hand, left hand, right foot, left foot, both feet, and tongue; many other tasks are also under research like those movements related to the elbow, fists, and fingers [3]. The MI-based BCI application involves clarification of the EEG signals and the determination of responses to those signals in real-time. Usually, analyzing EEG signals encountered the curse of dimensionality problem. People with neurological disease may find trouble in walking, speaking, and writing due to the lack of functioning of the motor control. Braincomputer interface (BCI) technology can help them to back to the quality of normal life[4]. A BCI application is described as the process of recording the brain's electroencephalogram (EEG) activity. After noise is removed from the recorded data, characteristics are retrieved and identified to perform a preset action (such as opening or shutting an artificial arm) [5]. Due to the vast size of the EEG data (1 second of EEG data may contain up to 250-time samples), feature extraction is required as the initial phase of the EEG classifier. The extraction of features is necessary since most classifiers perform matrix operations, and when a matrix is vast, it is referred to as an ill-conditioned matrix. The inverse of these matrices has significant numerical mistakes [6]. As a result, data compression is required. Typically, typical electroencephalographic characteristics are retrieved in time or frequency bands. The classifier's second stage is divided into two phases: training and recognition. In the training step, offline classification algorithms are trained using EEG data sets of known classifications [7], [8]. The unclassified EEG data is then sent into the classifier, which decides (determining which class the EEG samples belong to). The classification decision is subsequently communicated to the implementing hardware, which takes the appropriate action (moving an artificial arm or driving a wheelchair). Numerous feature reduction and classification algorithms have been developed for applications based on EEG-based Motor Imagery

This is an open access article under the terms of the Creative Commons Attribution License, which permits use, distribution and reproduction in any medium, provided the original work is properly cited.

(C) 2021 The Authors. Published by Iraqi Journal for Electrical and Electronic Engineering by College of Engineering, University of Basrah. 
(MI)[9], [10]. Multiple strategies for feature extraction have been considered in the literature[11], [12]:

1) Temporal characteristics.

2) The energy characteristics of the spectrum.

3) Statistical characteristics.

Numerous techniques are available for extracting time features, the most often utilized of which are: Eigen Value Decomposition (EVD), Independent Component Analysis (ICA), Principal Component Analysis (PCA), and Linear Discriminant Analysis (LDA) [13] and [5][14]. Because BCI applications demand real-time or near-real-time approaches, temporal features are the optimal candidate for computing time and complexity compared to other feature extraction techniques. Numerous studies studied the usage of various feature extraction techniques and discovered encouraging results. [15], [16] explored the feature matrix's small size problem, in which the number of time features is significantly greater than the number of channels. It achieves an accuracy of 84 percent while dealing with two-class problems.[17] achieved a maximum accuracy of 99 percent by combining CSP filtering with LDA. [18] demonstrated that the xDAWN method surpasses both ICA and PCA in terms of accuracy. [19] and [20] compared the accuracy of various spectral feature extraction techniques, including Power Spectral Density (PSD), time-frequency energy distributions, periodogram, spectrogram, and Morlet Wavelet. This work aims to develop an offline EEG-MI classifier using Support Vector Machines (SVM). It entails both of the preceding steps: feature extraction and classification. This article discusses all aspects of the SVM classifier's implementation as well as the relevant theoretical background. The following is the organization of the paper. Section 2 discusses the acquisition of brain signals. Section 3 discusses signal pre-processing. Section 4 discusses feature extraction and classification. The limits of LDA are then examined in Section III, along with possible solutions. A detailed description of the SVM classification algorithm is included. Section IV describes the setup technique as well as the recording process. The algorithm parameters are adjusted, and the resulting accuracy is illustrated in the figures [21], [22]. Figure 1 shown the stages of Braincomputer interface block diagram model.

\section{DATA ACQUISITION}

The initial layer of BCI systems is used to collect brain signals via invasive or non-invasive approaches. This is referred to as electroencephalography (EEG), when the brain's electrical activity is monitored in connection to its recorded in an experiment, which uses electrical spikes to transmit signals. Market- or clinical-grade instruments are being developed to detect these electrical impulses. The first consumer system for monitoring brain activity is the Emotiv Epoc, which includes 14 electrodes as shown in Fig. 2. Alternatively, NeuroSky's Epoc measures brain activity.

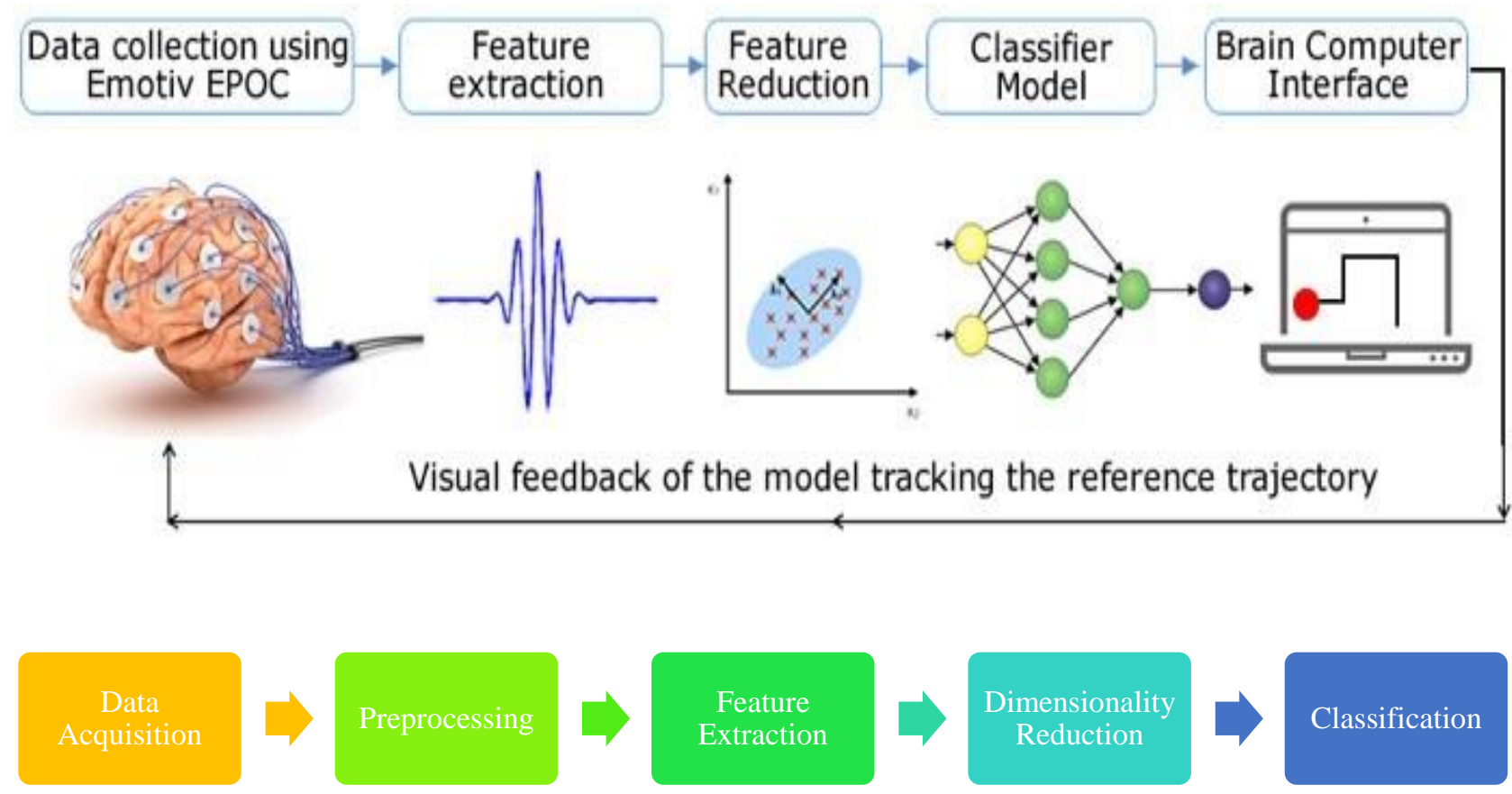

Fig. 1: The block diagram of the BCI-Based motor imagery system 


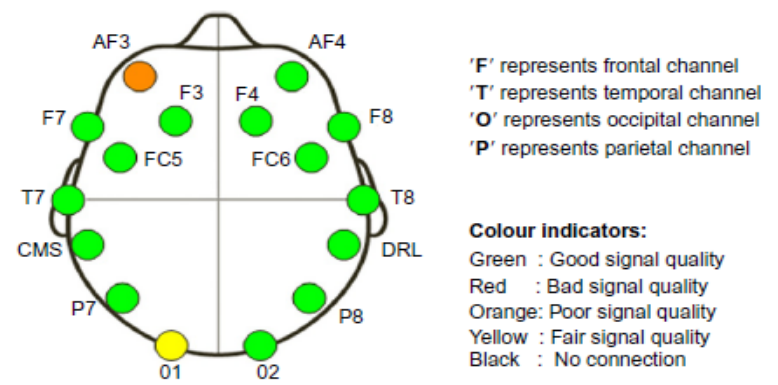

(a)

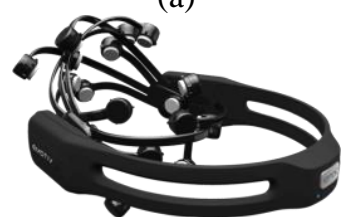

(b)

Fig. 2: EMOTIV EPOC headset

\section{Database Description}

The data for this article were collected using EMOTIV EPOC+. A data set contains four main tasks, one for each hand (class 1, class 2), one for each foot (class 3), and one for tongue pictures involving the tongue (class 4). Which mean the class one recorded from imagery moving left hand, class two from imagery right hand, class three from imagery moving foot and finally the class four from moving imagery the tongue. The recording sessions took place on two different days. Each session consisted of six runs separated by brief rest intervals. Each run had 48 trials (12 for each class). The timeframe of the experiment used to collect data is depicted in Fig. 3. The data contains of 42 subjects on two sessions every sessions contains of 16 seconds 8 with closed eyes and 8 with open eyes.

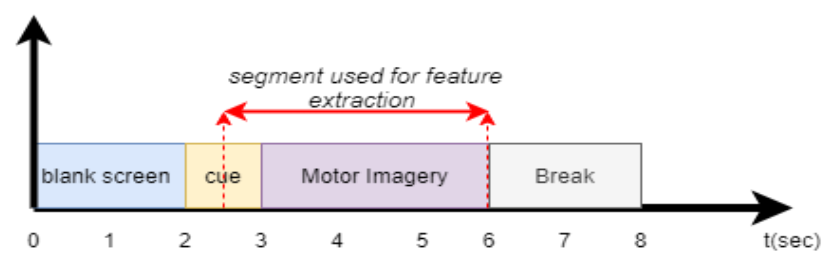

Fig. 3: Timing scheme of recorded EEG signal from the subjects and the segment used for feature extraction

\section{EEG Signal Pre-Processing:}

Following the signal acquisition, pre-processing reduces any noise or artifacts captured when acquiring the devices' signals. Among the undesirable signs are the following: Every time an electronic device is mounted, an inference is made. Specific muscular contractions result in EMG signals; eye movement or blinking results in the ocular artifact. The presence of undesired noise in the EEG recording will result in incorrect conclusions and skew the EEG findings' interpretation. As a result, several filters are utilized to eliminate noise from the signals. To begin, each channel was resampled to $128 \mathrm{~Hz}$ and filtered with a low pass filter in this article (Chebyshev Type II Lowpass filter, cutoff: $40 \mathrm{~Hz}$ ) as shown in Fig. 4 how shows the EEG signal before and after filtering.
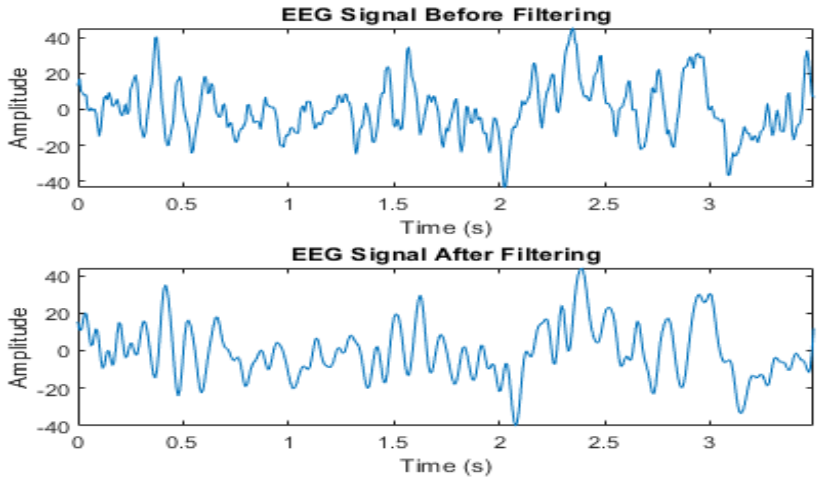

Fig. 4: EEG signal before and after filtering

\section{EEG SIGNAL EXTRACTION AND CLASSIFICATION:}

Due to the lack of discernible difference between distinct MI orders in the EEG signal time samples, it is impossible to classify time samples directly. The EEG time samples for two courses are depicted in Fig. 5. As can be observed, there is no discernible pattern of classification between the four classes. As a result, feature extraction is required, as the classification accuracy is dependent on it. The Fig. 5 shows scale plot of EEG time samples of single-channel for four classes.
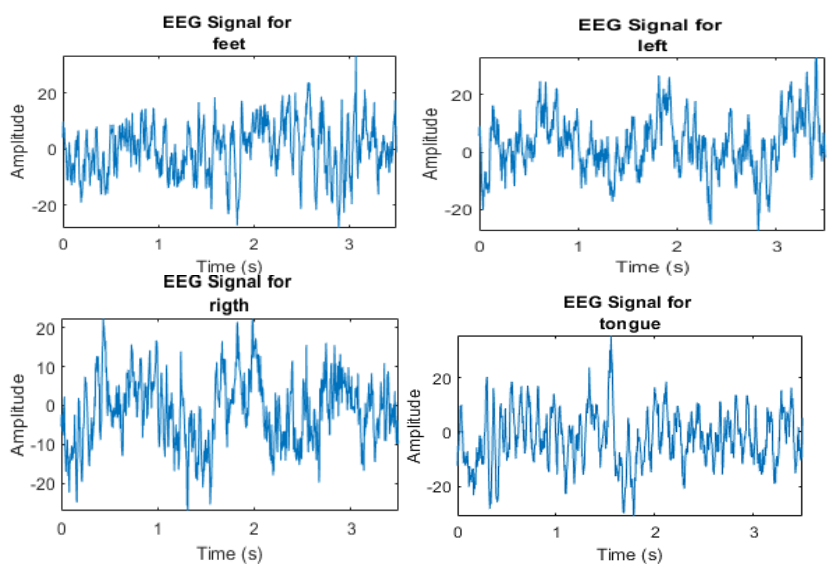

Fig. 5: Scale plot of EEG time samples of single-channel for four classes

\section{A. Feature Extraction}

Discrete Wavelet Transform with Maximum Overlap (MODWT) the relative tolerances are independent of the detail and approximation elements but can change as the details and approximation elements change. The wavelet approach was used to categorize theta, delta and theta patterns of brain activity in the experiment described in this work. Delta waves (intense concentration and sleep) are most frequently detected between 0 and $4 \mathrm{~Hz}$. Theta waves (4 to 8 $\mathrm{Hz}$ ) occur at extremely low frequencies during meditation and learning, whereas somewhat higher frequencies occur during sleep, research, and remembering. Alpha waves occur at frequencies between 8 and $12 \mathrm{~Hz}$ and are most evident when the brain is at rest. Beta waves, ranging from 12 to 32 $\mathrm{Hz}$, are generated when the brain actively engages in cognition or the external environment. $\mathrm{Mu}$ rhythm is 
generally between 8 and $13 \mathrm{~Hz}$ in frequency and is frequently associated with the rhythmic and non-rhythmic coordination of muscle groups. Before wave decomposition, it was essential to resample the original EEG signal to achieve 128 $\mathrm{Hz}$. It was conducted on both discrete wavelet transform (DWT), and complete overlap wavelet transform data (MODWT). A multistep approach was necessary for the analysis; in both cases, a Haar basis was used. Fig. 6 illustrates the decimation of the EEG into five distinct components (MODWT) using a maximum wavelet transform.

The Fig. 7 shows the EEG Signal decomposed into five bands the results of the bands shows in figure. It uses statistical metrics computed from each sub-band after wavelet decomposition over a window of 25 samples. Mean and variance, after sub-bans were obtained, the mean, variance, and Shannon entropy of each sub-bands were calculated and used as features. A total of 3 features $\times 5$ bands $\times 14$ channels $=210$ features were extracted for each trial as shown in Equations 1-2. Another measurement widely used in this type of experiment is the median, which is defined as the central value in a group of ordered data. The fourth function vectorization generator, which measures the uncertainty or complexity of a random signal, is defined as in Equation 3

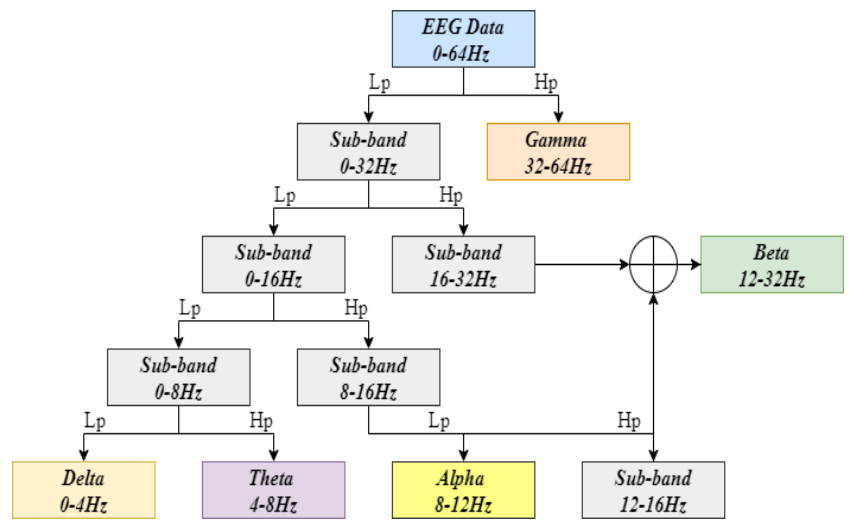

Fig. 6: EEG signal decomposed into five bands, with each significant sub-band highlighted.

$$
\begin{gathered}
\bar{x}=\frac{1}{n} \sum_{i=1}^{n} x_{i} \\
\sigma^{2}=\frac{1}{n} \sum_{i=1}^{n}\left(x_{i}-\bar{x}\right)^{2} \\
H(x)=\sum_{i=1}^{n} x_{i} \log _{2}\left(x_{i}\right)
\end{gathered}
$$
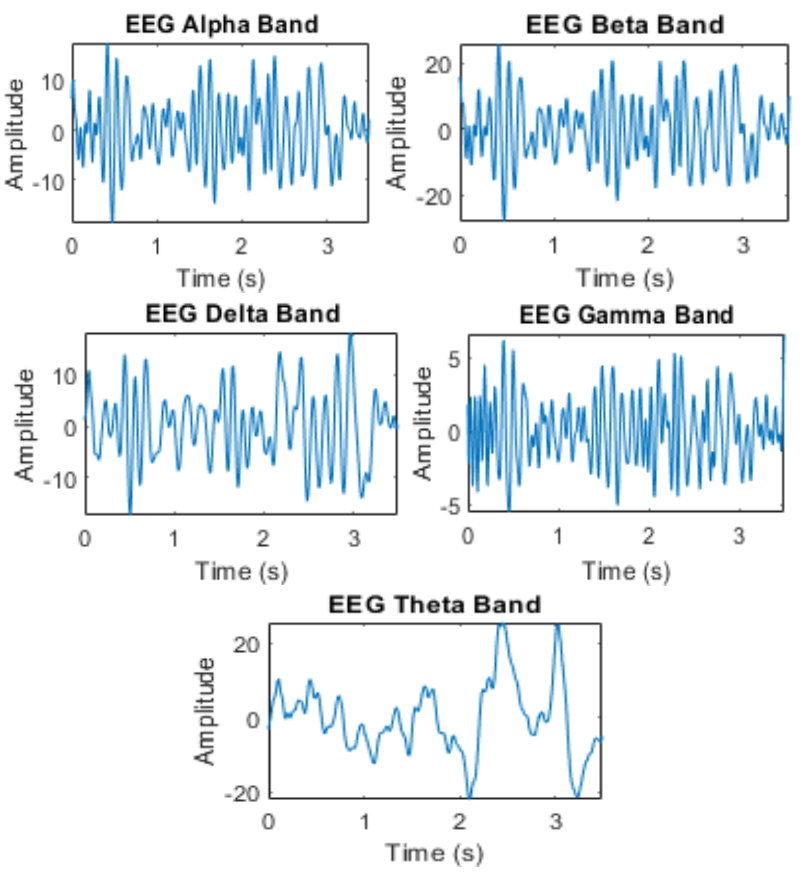

Fig. 7: MODWT Graphic band (Alpha, Beta, Delta, Gamma, Theta) after decomposition.

\section{B. Classification}

Several kernels were implemented as part of the support vector machines (SVMs) to compare each data type (e.g.g. Differentiate among different groupings of variables. Structural risk minimization consists of statistical learning with a technique that iterates on the data structure to construct a model and a probability distribution. Though linear discrimination is not possible in the definition of the algorithm does help us discriminate among various features in the input space is realized as features are mapped into a higher dimensional space that does not have this separation mapping can be performed using a linear or nonlinear algorithm, depending on the purpose of the used kernel. Instead of training decision trees, it first locates the class separators with the most significant margin between instances. Then, it finds the class separators and selects the ones that divide the classes with the slightest error. In various cases, the optimum hyperplane is expressed as a combination of several characteristics, known as the support vectors for the optimum. Here, we will use a straight-line, As shown in Fig. 8 the procedure of building SVM the standard, and the exponential functions for mappings to research the differences between them. 


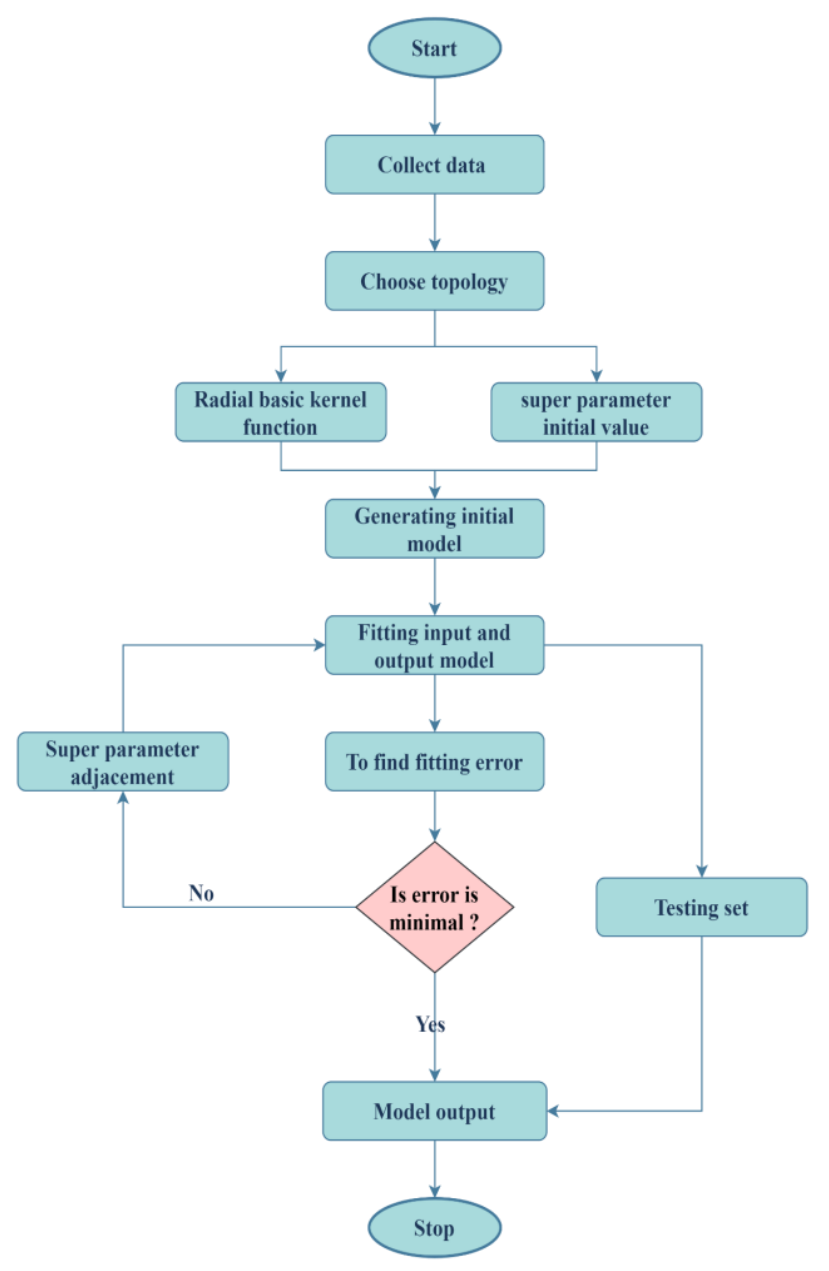

Fig. 8: The procedure of the SVM classifier.

\section{IMPLEMENTATION AND RESULTS}

The EEG dataset was collected using the EMOTIV EPOC headset, which provides 14 channels. The subject was asked to sit at rest state for 10 to 20 minutes before recording. This allows the relaxation of the brain activities in general and the accuracy of recorded EEG data. The subject is then asked to put both of his hands on a table in Front of him while his eyes are opened. Then, a cue is shown to him to imagine the movement of his right hand (Close and Opening). While he closes and opens his right hand repeatedly real movement and imagery movement, the EEG data is recorded for 5 seconds at a rate of 250 samples per second. Then repeat the operation for all three classes. Those trails are imported to MATLAB. Each trail consists of 640 samples. The trial recording is repeated until 120 trials are recorded and stored of all classes. These trials are named Class Right training. The same procedure is repeated for Class Left training samples, tongue class, and foot class. MATLAB 2020a environment is used to record, store and implement the SVM algorithm. After the training data is acquired, the features representing each class are extracted and stored as matrices. The subject then performed either left- or simple right-hand movement (closing and opening each hand) repeatedly for five seconds. The choice of which hand to move is random. During the subject session, the EEG signal is recorded at a sampling rate of 250 samples per second. This step is repeated for 120 trials with different random choices for either left- or right-hand tongue and foot of the subject. For each trial, the SVM algorithm extracts the features from the EEG data set, and those features are compared against the training features extracted during the training phase. According to the sign-in Table III, the winning class result is recorded. The accuracy of the algorithm is calculated as:

$$
\begin{aligned}
& \quad \begin{array}{c}
\text { Classification Rate }= \\
\text { True Positives }+ \text { True Negatives }
\end{array} \\
& \frac{T \text { True Positives }+ \text { False Positives }+ \text { True Negatives }+ \text { False Negatives }}{T P+T N+F P+F N}
\end{aligned}
$$$$
\text { Sensitivity }=\frac{\text { True Positives }}{\text { True Positives }+ \text { False Negatives }}
$$

$$
T P R=\frac{T P}{P}=\frac{T P}{T P+F N}=1-F N R
$$

$$
\text { Specificity }=\frac{\text { True Negatives }}{\text { False Positives }+ \text { True Negatives }}
$$

Positive predictive value $(P P V)=$

$$
\begin{gathered}
\frac{\Sigma \text { True positive }}{\Sigma \text { Predicted condition positive }}=\frac{T P}{T P+F P}=1-F D R \\
F N R=\frac{F N}{P}=\frac{F N}{F N+T P}=1-T P R \\
F D R=\frac{F P}{F P+T P}=1-P P V
\end{gathered}
$$

As it can be seen, the best channel accuracy occurs at channel 3 , which complies with the F3 sensor. This sensor is the nearest to the MI region in the brain, related to the EMOTIV EPOC headset (see Fig. 2). The regularization parameter ( $\eta$ ) selection plays a role in increasing the accuracy of the inverse of the matrix. Several values were tested for $\eta$ against accuracy, as shown in Fig. 5. The best accuracy occurs between $[0.85,0.9]$. Figure 6 shows the effect of window size (in samples) on the classification accuracy. It can be seen that a window size of approximately 1.2 seconds gives the best accuracy. In other words, the mean value for every 150 samples represents a particular EEG signal.

On the other hand, increasing the window size beyond 175 samples will decrease the accuracy. The reason for this accuracy degradation is that a larger window size will overlap with adjacent EEG samples. Therefore, the unique time feature characteristics will be destroyed. As a result, an EEG recording of 1.5 seconds is sufficient for LDA classification. Thus, the user does not have to repeat EEG emotion within a single trial. 


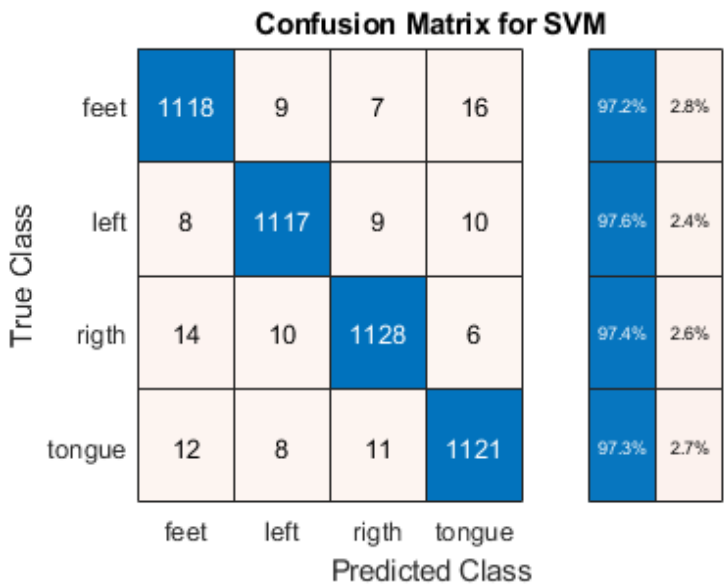

Fig. 9: Confusion matrix obtained using MODWT and SVM with cubic kernel

Table I

Comparison of different types of SVM classifier

\begin{tabular}{lcc}
\hline Classifier Type & $\begin{array}{c}\text { Train } \\
\text { Accuracy }\end{array}$ & $\begin{array}{c}\text { Test } \\
\text { Accuracy }\end{array}$ \\
\hline Fine Tree & $56.50 \%$ & $75.20 \%$ \\
\hline Linear Discriminant & $50 \%$ & $56.80 \%$ \\
\hline Naïve-Bayas Gaussian & $36.30 \%$ & $36.70 \%$ \\
\hline Quadratic SVM & $76.80 \%$ & $86.10 \%$ \\
\hline Cubic SVM & $92.60 \%$ & $97.20 \%$ \\
\hline Cosine K-NN & $57.30 \%$ & $53.50 \%$ \\
\hline Cubic K-NN & $54.60 \%$ & $51.50 \%$ \\
\hline Optimize Discriminant & $50 \%$ & $59.90 \%$ \\
\hline Optimize Naïve-Bayes & $70 \%$ & $74.80 \%$ \\
\hline Optimize Tree & $86.50 \%$ & $85.10 \%$ \\
\hline Linear SVM & $52.30 \%$ & $58.30 \%$ \\
\hline Ensemble K-NN & $82.40 \%$ & $76.80 \%$ \\
\hline
\end{tabular}

\section{CONCLUSION}

This article describes EEG Motor-Imagery BCI System Based on Maximum Overlap Discrete Wavelet Transform (MODWT) and Machine learning algorithm a study that proved how an EEG might identify electrical activity patterns associated with motor imagery using BCI devices. Maximum Overlap Discrete Wavelet analysis performed exceptionally well as a class separator. Using the defined features from the Maximum-Overlap Discrete classifier to identify specific subbands produced outstanding results. On average, the support vector machine achieved 98.81 percent accuracy. However, the support vector algorithm was only correctable to 97.77 percent when working on a nine-point kernel. Other movements in this research, which are currently in the robot control phase of development, entail simple ones.

\section{CONFLICT OF INTEREST}

The authors have no conflict of relevant interest to this article.

\section{REFERENCE}

[1] S. S. Abdulwahab, H. K. Khleaf, and M. H. Jassim, "A Systematic Review of Brain-Computer Interface Based EEG," Iraqi J. Electr. Electron. Eng., vol. 16, no. 2, pp. 110, Dec. 2020, doi: 10.37917/ijeee.16.2.9.

[2] B. Osalusi, A. Abraham, and D. Aborisade, "EEG Classification in Brain Computer Interface ( BCI ): A Pragmatic Appraisal," vol. 8, no. 1, pp. 1-11, 2018, doi: 10.5923/j.ajbe.20180801.01.

[3] A. Korik, R. Sosnik, N. Siddique, and D. Coyle, "Imagined 3D hand movement trajectory decoding from sensorimotor EEG rhythms," in 2016 IEEE International Conference on Systems, Man, and Cybernetics, SMC 2016 - Conference Proceedings, Feb. 2017, pp. 4591-4596, doi: 10.1109/SMC.2016.7844955.

[4] Z. M. Alhakeem and R. Ali, "Session to Session Transfer Learning Method Using Independent Component Analysis with Regularized Common Spatial Patterns for EEG-MI Signals," Iraqi J. Electr. Electron. Eng., vol. 15, no. 1, pp. 13-27, Jun. 2019, doi: 10.37917/ijeee.15.1.2.

[5] S. N. Abdulkader, A. Atia, and M. S. M. Mostafa, "Brain computer interfacing: Applications and challenges," Egypt. Informatics J., vol. 16, no. 2, pp. 213230, Jul. 2015, doi: 10.1016/j.eij.2015.06.002.

[6] S. Snyder and X. A. Shen, "A Review of Brain Signal Processing Methods," Int'l Conf. Biomed. Eng. Sci., vol. BIOENG'17, pp. 10-16, 2017.

[7] X. Zhang, L. Yao, X. Wang, W. Zhang, S. Zhang, and Y. Liu, "Know Your Mind: Adaptive Brain Signal Classification with Reinforced Attentive Convolutional Neural Networks," no. February, 2018, [Online]. Available: http://arxiv.org/abs/1802.03996.

[8] N. M. Firouz and S. Haghipour, "A Survey on EEG Signal Classification with Neural Network for Brain Computer Interface Applications," Int. J. Comput. Inf. Technol., vol. 4, no. 2, pp. 34-42, 2016.

[9] R. Abiri, S. Borhani, E. W. Sellers, Y. Jiang, and X. Zhao, "A comprehensive review of EEG-based braincomputer interface paradigms," J. Neural Eng., vol. 16, no. 1, p. 011001, Feb. 2019, doi: 10.1088/17412552/aaf12e.

[10] N. Padfield, J. Zabalza, H. Zhao, V. Masero, and J. Ren, "EEG-based brain-computer interfaces using motorimagery: Techniques and challenges," Sensors (Switzerland), vol. 19, no. 6, pp. 1-34, 2019, doi: 10.3390/s19061423.

[11] D. Bansal and R. Mahajan, EEG-Based BrainComputer Interfacing (BCI). Elsevier Inc., 2019.

[12] R. A. Ramadan and A. V. Vasilakos, "Brain computer interface: control signals review," Neurocomputing, vol. 223, no. October 2016, pp. 26-44, Feb. 2017, doi: 10.1016/j.neucom.2016.10.024.

[13] J. D. R. Millán et al., "Combining brain-computer interfaces and assistive technologies: State-of-the-art and challenges," Frontiers in Neuroscience, vol. 4, no. SEP. 2010, doi: 10.3389/fnins.2010.00161.

[14] H. Zhou, "Principles and Applications in Brain Computer Interfaces," no. March, pp. 1-13, 2018.

[15] N. Tiwari, D. R. Edla, S. Dodia, and A. Bablani, "Brain 
computer interface: A comprehensive survey," Biol. Inspired Cogn. Archit., vol. 26, no. October, pp. 118-129, 2018, doi: 10.1016/j.bica.2018.10.005.

[16] V. G. Sangam, S. B. M, S. S. Raman, A. S. Lakshmi, P. A. Murthy, and M. Faizan, "Electroencephalogram ( EEG ), its Processing and Feature Extraction," no. June, 2020, doi: 10.17577/IJERTV9IS060814.

[17] B. Rivet, A. Souloumiac, V. Attina, and G. Gibert, "xDAWN Algorithm to Enhance Evoked Potentials: Application to Brain-Computer Interface," IEEE Trans. Biomed. Eng., vol. 56, no. 8, pp. 2035-2043, 2009, doi: 10.1109/TBME.2009.2012869.

[18] N. Brodu, F. Lotte, and A. Lécuyer, "Comparative study of band-power extraction techniques for Motor Imagery classification," in IEEE SSCI 2011 - Symposium Series on Computational Intelligence - CCMB 2011: 2011 IEEE Symposium on Computational Intelligence, Cognitive Algorithms, Mind, and Brain, 2011, pp. 95-100, doi: 10.1109/CCMB.2011.5952105.

[19] A. Khosla, P. Khandnor, and T. Chand, "A comparative analysis of signal processing and classification methods for different applications based on EEG signals," Biocybern. Biomed. Eng., vol. 40, no. 2, pp. 649-690, 2020, doi: 10.1016/j.bbe.2020.02.002.

[20] J. Meng, S. Zhang, A. Bekyo, J. Olsoe, B. Baxter, and B. He, "Noninvasive Electroencephalogram Based Control of a Robotic Arm for Reach and Grasp Tasks," Sci. Rep., vol. 6, p. 38565, Dec. 2016, doi: 10.1038/srep38565.

[21] A. Al-saegh, "Comparison of Complex-Valued Independent Component Analysis Algorithms for EEG Data," Iraq J. Electr. Electron. Eng., vol. 15, no. 1, pp. 112, Jun. 2019, doi: 10.37917/ijeee.15.1.1.

[22] A. Al-Saegh, S. A. Dawwd, and J. M. Abdul-Jabbar, "Deep learning for motor imagery EEG-based classification: A review," Biomedical Signal Processing and Control, vol. 63. Elsevier Ltd, Jan. 01, 2021, doi: 10.1016/j.bspc.2020.102172.

[23] H. Wang, Y. Li, J. Long, T. Yu, and Z. Gu, "An asynchronous wheelchair control by hybrid EEG-EOG brain-computer interface," Cogn. Neurodyn., vol. 8, no. 5, pp. 399-409, Oct. 2014, doi: 10.1007/s11571-014-9296-y.

[24] S. Selim, M. Tantawi, H. Shedeed, and A. Badr, "Reducing execution time for real-time motor imagery based BCI systems," in Advances in Intelligent Systems and Computing, Oct. 2017, vol. 533, pp. 555-565, doi: 10.1007/978-3-319-48308-5_53.
[25] Q. Huang, Z. Zhang, T. Yu, S. He, and Y. Li, “An EEG/EOG-Based Hybrid Brain-Computer Interface: Application on Controlling an Integrated Wheelchair Robotic Arm System," Front. Neurosci., vol. 13, p. 1243, Nov. 2019, doi: 10.3389/fnins.2019.01243.

[26] S. Selim, M. M. Tantawi, H. A. Shedeed, and A. Badr, "A CSP\AM-BA-SVM Approach for Motor Imagery BCI System," IEEE Access, vol. 6, pp. 49192-49208, Aug. 2018, doi: 10.1109/ACCESS.2018.2868178.

[27] N. Padfield, J. Zabalza, H. Zhao, V. Masero, and J. Ren, "EEG-based brain-computer interfaces using motorimagery: Techniques and challenges," Sensors (Switzerland), vol. 19, no. 6, p. 1423, Mar. 2019, doi: 10.3390/s 19061423.

[28] R. D. Roy Amit Konar N Tibarewala, "CONTROL OF ARTIFICIAL LIMB USING EEG \& EMG-A REVIEW.”

[29] M. Kołodziej, A. Majkowski, and R. J. Rak, "A new method of EEG classification for BCI with feature extraction based on higher order statistics of wavelet components and selection with genetic algorithms," in Lecture Notes in Computer Science (including subseries Lecture Notes in Artificial Intelligence and Lecture Notes in Bioinformatics), 2011, vol. 6593 LNCS, no. PART 1, pp. 280-289, doi: 10.1007/978-3-642-20282-7_29.

[30] D. R. Edla, M. F. Ansari, N. Chaudhary, and S. Dodia, "Classification of Facial Expressions from EEG signals using Wavelet Packet Transform and SVM for Wheelchair Control Operations," in Procedia Computer Science, Jan. 2018, vol. 132, pp. 1467-1476, doi: 10.1016/j.procs.2018.05.081.

[31] H. Yuan, A. Doud, A. Gururajan, and B. He, "Cortical imaging of event-related (de)synchronization during online control of brain-computer interface using minimum-norm estimates in frequency domain," IEEE Trans. Neural Syst. Rehabil. Eng., vol. 16, no. 5, pp. 425431, Oct. 2008, doi: 10.1109/TNSRE.2008.2003384.

[32] O. Attallah, J. Abougharbia, M. Tamazin, and A. A. Nasser, "A bci system based on motor imagery for assisting people with motor deficiencies in the limbs," Brain Sci., vol. 10, no. 11, pp. 1-25, Nov. 2020, doi: 10.3390/brainsci10110864.

[33] J. Kevric and A. Subasi, "Comparison of signal decomposition methods in classification of EEG signals for motor-imagery BCI system," Biomed. Signal Process. Control, vol. 31, pp. 398-406, Jan. 2017, doi: 10.1016/j.bspc.2016.09.007.

TABLE II

Performance characteristics of SVM Classifier.

\begin{tabular}{ccccccccc}
\hline \multirow{2}{*}{ Classes } & \multicolumn{2}{c}{ TPR } & \multicolumn{2}{c}{ FNR } & \multicolumn{2}{c}{ PPV } & \multicolumn{2}{c}{ FDR } \\
& True positive rate & False-negative rate & Positive predictive value & False discovery rate \\
\cline { 2 - 9 } & Train & Test & Train & Test & Train & Test & Train & Test \\
\cline { 2 - 9 } Foot & $94.8 \%$ & $92.1 \%$ & $5.2 \%$ & $7.9 \%$ & $99.1 \%$ & $91.7 \%$ & $0.9 \%$ & $8.3 \%$ \\
\hline Left hand & $96.8 \%$ & $92.0 \%$ & $3.1 \%$ & $8.0 \%$ & $94.8 \%$ & $93.7 \%$ & $5.2 \%$ & $6.3 \%$ \\
\hline Right hand & $97.8 \%$ & $92.3 \%$ & $2.2 \%$ & $7.7 \%$ & $99.1 \%$ & $90.1 \%$ & $0.9 \%$ & $9.9 \%$ \\
\hline Tongue & $99.1 \%$ & $92.4 \%$ & $0.9 \%$ & $7.6 \%$ & $95.8 \%$ & $93.5 \%$ & $4.2 \%$ & $6.5 \%$ \\
\hline
\end{tabular}


Table III

Comparison result with previous works of detection and classification of EEG-based Motor-Imagery

\begin{tabular}{|c|c|c|c|c|c|c|}
\hline Article & Year & Application & $\begin{array}{c}\text { Feature } \\
\text { Extraction }\end{array}$ & Classifier & Disadvantages & $\begin{array}{c}\text { Accuracy } \\
(\%)\end{array}$ \\
\hline [23] & 2014 & Limb Motor Task & $\begin{array}{c}\text { Spatial- } \\
\text { frequency } \\
\text { temporal patterns }\end{array}$ & SRC & $\begin{array}{c}\text { High } \\
\text { computational cost }\end{array}$ & 75.46 \\
\hline [24] & 2017 & Limb Motor Task & RMS & LDA & $\begin{array}{l}\text { Time-domain } \\
\text { features not } \\
\text { suitable for } \\
\text { analysis EEG } \\
\text { datasets } \\
\end{array}$ & 78.77 \\
\hline [25] & 2019 & $\begin{array}{c}\text { Integrate a } \\
\text { wheelchair and an } \\
\text { artificial limb }\end{array}$ & CSP & SVM & $\begin{array}{l}\text { CSP suffers from } \\
\text { degradation in } \\
\text { performance in } \\
\text { case of non- } \\
\text { Gaussian } \\
\text { distributions }\end{array}$ & 80 \\
\hline [26] & 2018 & Limb Motor Task & CSP & SVM & $\begin{array}{l}\text { Required high } \\
\text { input channels }\end{array}$ & 85.01 \\
\hline [27] & 2019 & Limb Motor Task & CSP & MDRM & $\begin{array}{l}\text { Required high } \\
\text { input channels }\end{array}$ & 86.13 \\
\hline [28] & 2011 & $\begin{array}{l}\text { Control an artificial } \\
\text { limb }\end{array}$ & DWT & $\begin{array}{l}\text { LDA } \\
\text { QDA } \\
\text { KNN }\end{array}$ & $\begin{array}{l}\text { DWT gives lower } \\
\text { frequency } \\
\text { resolution than } \\
\text { WPD }\end{array}$ & 86.9 \\
\hline [29] & 2011 & $\begin{array}{c}\text { Control artificial } \\
\text { limb by paralyzed } \\
\text { patients }\end{array}$ & $\mathrm{HOS}+\mathrm{DWT}$ & LDA & $\begin{array}{l}\text { DWT gives lower } \\
\text { frequency } \\
\text { resolution than } \\
\text { WPD }\end{array}$ & 89.5 \\
\hline [30] & 2018 & control wheelchairs & WPD & SVM & $\begin{array}{c}\text { High } \\
\text { computational cost }\end{array}$ & 90.68 \\
\hline [31] & 2008 & Limb Motor Task & FT & MNFD & $\begin{array}{l}\text { FT not suitable for } \\
\text { analysis of EEG } \\
\text { data }\end{array}$ & 90.89 \\
\hline [32] & 2020 & $\begin{array}{c}\text { Real-time } \\
\text { wheelchair control }\end{array}$ & FFT & FNN & $\begin{array}{c}\text { FFT not suitable } \\
\text { for analysis EEG } \\
\text { dataset }\end{array}$ & 92.0 \\
\hline [33] & 2017 & Limb Motor Task & DWT + WPD & KNN & $\begin{array}{l}\text { KNN suffers from } \\
\text { the curse of } \\
\text { dimensionality }\end{array}$ & 92.8 \\
\hline $\begin{array}{l}\text { The } \\
\text { proposed } \\
\text { work }\end{array}$ & 2021 & Limb motor tasks & $\begin{array}{c}\text { MODWT+ } \\
\text { Statical features }\end{array}$ & SVM & & 97.8 \\
\hline
\end{tabular}

